\title{
Physical Constants as Identifiers of Modern Universal Laws of Nature
}

\author{
Orli Dahan*
}

Received: 18 January 2019 / Accepted: 3 December 2019

Abstract: I argue that in modern algebraic-formulated science the 'physical constant' can be understood, for practical purposes, as an 'identifier' of a universal law of nature. This identifying role is possible because the concept of 'physical constant' fulfills the same need for universality, stability, and fundamentality (as universal laws) for increasing the epistemic value of a scientific theory. This can be demonstrated in two different ways. The first involves a thought experiment envisioning science without physical constants, which appears to be a science of local and particular laws. The second is the observation that physical constants mostly emerge as components in an algebraic formulation of universal laws, but not in the algebraic formulation of particular laws. This observation about the link between physical constants and universal laws of nature, if correct, makes two contributions. First, it clarifies, at least partially, the ambiguity in the use (and the absence) of the concept 'law' in contemporary science. Second, it can help in distinguishing between a universal law and a particular law, while avoiding one of the abiding philosophical problems regarding laws of nature - the problem of the ceteris-paribus criterion for a generalization.

Keywords: Law of nature; universal law; particular law; physical constant; ceteris-paribus generalization; strict generalizations.

* Tel-Hai College

Faculty of Humanities and Social Sciences, Tel-Hai College, Upper Galilee, 12208, Israel

$\bowtie$ orlydah@telhai.ac.il

() The Author. Journal compilation (C) The Editorial Board, Organon F.

This article is distributed under the terms of the Creative Commons Attribution-NonCommercial 4.0 International Public License (CC BY-NC 4.0). 


\section{Introduction}

Physical constants are essential to current physical theories; they are values in many physics equations. Such constants include the velocity of light $(c)$, Newton's constant of gravitation $(G)$, and Planck's constant $(h) .{ }^{1}$ Physical constants are dimensional quantities with two fixed parameters: they are both constant at every point in space and have a constant value in time. In fact, physical constants have become so important to modern science, that it seems that every new grand physical theory introduces a new constant. For example, string theory and its coupling constant (Greene 2003, 303-06), or GRW in quantum mechanics and its new collapse constant (Frigg 2009).

In this paper, I argue for a conceptual link between modern physical constants and the concept of universal laws of nature. Defining 'law of nature' ('what is it to be a law?') is not an easy task. Definitions vary widely among scientists and philosophers of science and are dependent upon one's metaphysical commitments (see Caroll 2016). However, choosing a side in this age-old debate will not contribute to the primary goal here. So, for the purpose of this paper, I choose to define superficially the term 'universal law' via negation - contrasting it with the term 'particular law.' By 'universal law,' I mean a general statement that governs a class of phenomena and is universal in the sense that it has no exceptions: it appears to be applied everywhere in the universe in the same way. In this sense it is absolute and stable. A straightforward example of such a law is the law of inertia. It is universal in the sense that it governs all bodies in the world. In contrast, by 'particular law' I mean a general statement that governs a class of phenomena, but is particular in the sense that it cannot be applied

1 About 20 physical constants are known today, including, also, the rest mass of an electron $\left(\mathrm{m}_{\mathrm{e}}\right)$, Avogadro constants $\left(\mathrm{N}_{\mathrm{A}}\right)$, and Boltzmann's constant $\left(\mathrm{k}_{\mathrm{B}}\right)$.

2 Definition via negation is popular in the field of philosophy of mind, where there is a long-standing discussion regarding how to define the concept 'physical' (see Crane and Mellor 1990). The most accepted way currently to solve this dilemma is 'via negativa.' This solution proposes to render the term 'mental' as fundamental and to characterize the physical as 'non-mental,' i.e. defining the term 'physical' negatively (Dahan 2019; Fiorese 2016; Prelević 2017). 
everywhere in the universe. In this sense it is conditional (rather than absolute). A good example is natural selection. Natural selection is a key mechanism of evolution (the change in the heritable traits characteristic of a population over generations), but it is not universal, for it is applicable only to organisms and not to non-vital objects. Another example from physics itself is Ohm's law, which is conditional, for is not applicable in extreme temperatures, or in some electrical components such as semiconductors. ${ }^{3}$

Caroll (2016) considers whether philosophy can advance beyond the dispute over laws of nature given the question of whether lawhood is a necessary component of scientific theories. We can see that in current physics, the status of the nomic term "law of nature" has practically fallen from grace, as opposed to (for example) its status at the time of the scientific revolution (see Ruby 1986; Shapin 1996; Zilsel 1942). ${ }^{4}$ For example, we still use the term "the laws of motion," or "Newton's second law," but we do not refer to the equations of quantum mechanics (QM) as 'laws.' Are the uncertainty principle or Schrödinger equation laws of QM? Are they universal laws in the meaning that "Newton's second law" was considered a universal law? According to Reutlinger et al. (2017), the Schrödinger equation clearly fits the traditional concept of a universal law. Either way, it can be said that the term "law" is absent from current, formal statements of modern theoretical physics. Indeed, according to Milton (1981), the discoveries of modern physics since Maxwell (1831-1879) have for the most part been expressed in terms of principles and equations rather than laws.

3 This particular example will be discussed in Section 3.

4 According to Shapin (1996), sixteenth and seventeenth century scientists and philosophers disputed the question whether science must be a mathematically formulated binding set of laws of nature, or whether mathematical representations could actually capture the contingencies and complexities of real natural processes (Shapin 1996, 58-59). Robert Boyle (1627-1691), for example, was uncomfortable with the common understanding of the concept 'laws of nature,' and repeatedly cautioned that it should be used carefully-moving from observed regularities to laws of nature obviates the factor of God's pleasure, power, and willing (Shapin 1996, 150). This discussion is evidence that the concept 'law of nature' was central at that time. 
I carefully assume that maybe the partial absence of 'laws' in theoretical physics is due to the existence in modern physics of other underlying principles, such as symmetries and super-symmetries, which are not laws but rather constraints upon phenomena, laws, and theories. ${ }^{5}$ Such a constraint can be seen in Fermat's principle. This principle is not a law, but serves as a guiding principle in the formulation of physical laws using the calculus of variations. ${ }^{6}$ Other constraints are symmetry principles in physics, such as Noether's theorem, which states that every differentiable symmetry of the action of a physical system has a corresponding conservation law (Rosen 1989). ${ }^{7}$

Nonetheless, even in current science we do use the term 'law.' However, this term, it seems, is used in science without distinction between universal law and particular law. This also happens in the field of physics itself. For example, we use the term 'law' in order to refer to "the law of gravitation," which is considered a universal law, and we use the term 'law' to refer to "Ohm's law," which is not considered a universal law. The same can be said with regard to the "ideal gas law" (universal law) and the "Boyle-Mariotte law," a special case of the ideal gas law, hence not a universal law. As noted, the ambiguity is also in the other direction: laws in fundamental physics are not called 'law' anymore. For example, the Schrödinger equation in QM is called an 'equation' even though it is one of the important laws of QM. This ambiguity in the use (and the non-use) of the term 'law' in modern physics calls for explanation and clarification, for it is possible that in

5 The relationship between laws and constraints in physics is worth examination. For example, can a law be a specific type of constraint? This discussion, however, is beyond the scope of this paper, and the answer to this question will not affect the arguments presented here.

${ }_{6} \quad$ For an empirical example see (Westphal et al. 2002).

7 Rosen (1989), for example, shows that when following the definition of symmetry as invariance under transformation, both reproducibility and predictability are kinds of symmetry. And since reproducibility and predictability are the two most fundamental principles of science - he argues that symmetry not only serves within science but actually lies at its very foundation. In my opinion, Rosen's approach is as an example for the falling from grace of the term "law of nature" in the realm of theoretical physics during the $20^{\text {th }}$ and $21^{\text {st }}$ centuries, and the rise of new terms (other than 'universal law of nature') that are considered fundamental and more essential. 
algebraic-formulated fundamental science we use the term 'law' vaguely, or sometimes do not use it at all. ${ }^{8,9}$

The straightforward conceptual link between the two concepts - 'law of nature' and 'physical constant' - is obvious: both are connected to fundamentality, universality, and stability. However, it is also clear that these terms refer to different entities with different logical formulations - a necessary relation in the case of laws of nature, and a contingent quantity in the case of physical constants. Nevertheless, from the introduction it follows that there are (at least) two main reasons that a philosophical investigation of the link between physical constants and laws of nature is needed. The first reason is that the latter term is still in use, yet its use is typically ambiguous. The second reason is that a new concept appeared in the physics of the late $19^{\text {th }}$ century - the 'physical constant' - which became an important component of physical theories, ${ }^{10}$ and has a straightforward, conceptual link to the senior concept 'universal law of nature.'

In this paper, I propose a method for distinguishing between universal and particular 'law' using the concept of physical constants. I will suggest the observation that physical constants generally emerge as components in the algebraic formulation of universal law, but not in the algebraic formulation of particular laws. Thus, I will investigate the hypothesis that physical constants, although clearly referring to different entities, can be used

8 In fact, it would be interesting to check whether all laws termed specifically as 'laws' in modern physics are older than the $20^{\text {th }}$ century (before the age of physical constants) while newer 'laws' are not called laws anymore (such as in QM and relativity). If true, the question is then whether the source of this change is the ambiguity in the concept 'law,' the shift to algebraical formulations in physics, or another reason. However, analysis of the historical reasons for the use and non-use of the term 'law' in modern physics is beyond the scope of this paper.

9 A note is needed here: I am primarily making a sociological-historical claim about how scientists talk about 'laws,' 'constraints,' and 'constants,' and not a philosophical claim about how I think scientists should talk about these terms.

10 Newton's constant of gravitation $(G)$ was clearly the first universal physical constant to appear, but its value was empirically calculated only at the end of the $18^{\text {th }}$ century by Henry Cavendish (1731-1810; Clotfelter 1987). Furthermore, the constant was given a symbol (the letter $G$ ) only in 1873, 186 years after Newton's Principia (in Cornu and Baille 1873). 
for testing the way we think of a certain phenomenon, field, branch of science, or even science in general: whether a phenomenon is fundamental in the sense that it is governed by universal laws, or whether it is (only) local and governed by particular laws. Using a thought experiment, I suggest that in modern science, the role of physical constants differs in theories that have differing scopes.

In Section 2, I present my thought experiment: what would physical science look like if we came to understand that the fundamental physical constants are not constants at all and, in fact, that there are no such stable physical quantities. The thought experiment is a framework for thinking about one of the functions of physical constants in science, and for suggesting my reflections on the conceptual link between constants and universal laws. In Section 3, I give examples to further the following objectives: (a) to support my hypothesis that physical constants emerge as components in the algebraic formulation of universal laws, but not in the algebraic formulation of particular laws; (b) to further clarify my philosophical principle that physical constants are identifiers of universal laws, and the limitations of this principle (i.e. only in algebraic formulated science); and (c) to demonstrate how my principle can help us distinguish between a universal law and a particular law, while avoiding one of the abiding philosophical problems regarding laws of nature (the ceteris-paribus criterion for a generalization). In Section 5, I conclude this philosophical investigation and in Section 6, I discuss possible implications for the ideas presented.

\section{The thought experiment: modern physics without physical constants}

To set the ground for my thought experiment, a short analysis of the concept "physical constant" is in place. What is it to be a physical constant? Or, stated differently: what are the necessary conditions for a physical quantity to gain the status of a physical constant? The straightforward answer is that this quantity must be unchangeable in space and in time, in the meaning of 'steady.' This simple definition also applies to one of the features of a universal law of nature, which is supposed to govern all phenomena at 
all points in space, and at all times. In fact, this is, I think, the meaning of 'universality.' So, in this simple matter of superficial definitions, we can see that there might be a connection between the two concepts.

Now, suppose we were to discover in the near future that all fundamental physical constants are not constant at all. More precisely, we learn that we were wrong to believe that these physical quantities are fixed quantities: either we come to understand that the constants are not constants (for example we will find out that the speed of light in a vacuum is not the maximum speed of massless particles), or that they do not exist at all (something like the mistake Einstein made with the cosmological constant). ${ }^{11}$ The question this thought experiment is addressing, which I will answer throughout this paper, is the following: how does this imaginary scenario affect physics?

To answer this question, we first need to ask what makes a physical constant, constant, and how do we come to understand that a certain physical quantity is not really a constant physical quantity?

In fact, the thought experiment I propose is not so far-fetched, for there are various discussions in the literature regarding this possibility. ${ }^{12}$ Moreover, the reverse of this thought experiment is basically how we give a specific dimensional physical quantity its status as a physical constant, for in some cases, we come to understand that a certain dimensional physical quantity is universally constant after initially thinking it a 'particular' constant. The most straightforward example is the speed of light $c$ : when it was first measured, it was only a characteristic of a particular system. ${ }^{13}$ However, with the development of classical electromagnetism, it became a characteristic of a class of physical phenomena (electromagnetic phenomena). Only with the

11 For more about Einstein's mistake regarding the cosmological constant $(\Lambda)$ see (Harvey and Schucking 2000). For a review of the basic physics and astronomy of the subject and the history of this idea see (Peebles and Ratra 2003).

12 For example, Mangano et al. (2015) consider the possibility that the Planck constant is a time dependent quantity, undergoing random Gaussian fluctuations around its measured constant mean value. Support for this possibility is derived from Dirac's idea that fundamental constants are dynamic variables and from conjectures on quantum structure of space-time at small distances.

13 For the history of measuring the speed of light see (Romer and Cohen 1940). 
discovery of special relativity did the speed of light become a physical constant (Lévy-Leblond 1979).

As noted above, physical constants have two fixed parameters: they are dimensional quantities that are both constant at every point in space and have a constant value in time. But are these two parameters necessary for the constants to be universal in nature? According to Uzan (2011), "Any [physical] constant varying in space and/or time would reflect the existence of an almost massless field that couples to matter. This will induce a violation of the universality of free fall. Thus, it is of utmost importance for our understanding of gravity and of the domain of validity of general relativity to test for their constancy."

In principle, however, it is conceivable to have a physical constant that changes in time, but stays fixed for all points in space. For example, if we were to discover that $G$, the universal gravitational constant, changes slightly in time (periodically or linearly), and this change in time has some fixed regularity that is, in principle, determinate, quantifiable and predictable - then $G$ is still a physical constant because it is constant at any point of the universe at a certain time - it is not local.

However, if we were to discover that $G$ 's value on earth is not the same as on one of Jupiter's moons, then it appears that we will have to deprive $G$ of its status as a physical constant. And why? Because it is obvious that what is really unique about the physical constants is that they are constant at every point in space at a certain time. Now, if we discover that $G$ is not a constant, the true implication of this discovery will be that the law of gravitation is not universal, as we thought it was. In this case, we will have to accept the fact that this law is a local law, for it does not govern the phenomenon of free fall on one of Jupiter's moons in the same manner it governs this phenomenon on earth.

In order to examine the implications for science of the elimination of physical constants, must I check all (around) 20 physical constants known today? I believe the answer is negative and that it is sufficient for the purpose of this thought experiment to consider only the three fundamental constants, $G, c$ and $h .^{14}$

14 In fact, Matsas et al. (2007) argue that even these three can be reduced to just two fundamental constants (they prefer eliminating $G$, but apparently any of the 
Let us now consider the other two fundamental physical quantities: the speed of light $(c)$ and the Planck constant $(h)$. As noted above, initially the speed of light was not considered a universal constant, so it is simple to imagine how reversion to this understanding will affect current physics. If, in the near future, we come to realize that $c$ is not a constant quantity, ${ }^{15}$ this 'constant' will probably revert to its former status as a characteristic of a particular system, or a characteristic of electromagnetic phenomena. But how will that affect what we think of electromagnetic phenomena? Will it still be considered a fundamental phenomenon? I hypothesize that if we discover that the other constants relevant to electromagnetic phenomena, such as the Planck constant $(h)$ and electrostatic constant $\left(k_{e}\right)$, are not constants, then the answer is affirmative: we will probably consider that electromagnetic phenomena are not universal. They may be considered local phenomena, or perhaps candidates for a special case of more fundamental phenomena. Consequently, the laws of electromagnetics will be considered particular laws and not universal laws. And what would that do to relativity theory? If $c$ is no longer a physical quantity independent of any frame of reference - then it seems that relativity theory can no longer be considered a fundamental theory. Relativity theory cannot be a theory describing the whole universe, for it is not universal anymore.

The same point can be made regarding the Planck constant $h$ : this constant is the most central to quantum mechanics. If we come to understand that this constant is not fixed at all places in the universe - it seems that we will have to construct a completely new fundamental physics. ${ }^{16}$

three is a candidate), and that their proof is model independent. This also indicates that the long search of fundamental science for a universal law of nature (an underlying law that governs all there is) has shifted in contemporary science. In physics today, 'laws' are just another component of a scientific theory, and it even seems that the main focus of reduction is not laws, as it once seemed to be.

15 For example, we will find out that $c$ is not the finite speed of massless particles on Jupiter, and that it seems that only on Jupiter and beyond, the finite speed is $350,000 \mathrm{~km} / \mathrm{s}$.

16 No one really knows how this 'new physics' will look, if we come to understand that the constants are not constants. As Barrow and Webb (2005) conclude: "The constants are a tantalizing mystery. Every equation of physics is filled with them, and they seem so prosaic that people tend to forget how unaccountable their values 
For example, Lizzi et al. (2016) discuss the possibility that the fundamental Planck constant has stochastic fluctuations, set by a possible quantum structure of space-time. It is worth noting that although being time dependent is a nontrivial affair for fundamental dimensional quantities, this possibility is being discussed currently in the field of theoretical physics. However, I have not found discussions in the literature of physical science regarding the possibility that this constant (or the other fundamental constants) may not be constant in space. This possibility would change the quantity from universal to local and undeserving of the status of "physical constant;' yet, determinable regular changes in time while remaining constant in space would not deprive $h$ of its characterization as a physical constant.

Since modern day universal laws of physics are essentially these universal equations, if we come to understand that there are no true physical constants in fundamental science, it seems that we will have to give up universality within the equations themselves: we will have only local laws. This will not necessarily affect other components of physical theories (such as underlying principles). However, laws that are expressed in modern science in the form of algebraic equations will have to be regarded as local laws only.

This thought experiment demonstrates that what is unique about physical constants is that they are fixed for all points in space. If we waive that requirement - then what we get is locality, which is (in this context) the opposite of universality. Thus, it seems to me that current universal laws whose physical constants are not strictly universal (the value can remain in the equation as a sort of 'local quantity;' as with $c$ before general relativity) actually become particular laws. Hence, I draw the following hypothesis:

i. Algebraic science (like ours) without physical constants is the science of particular laws that govern (in the best case) only a class of physical phenomena.

are. Their origin is bound up with some of the grandest questions of modern science, from the unification of physics to the expansion of the universe. They may be the superficial shadow of a structure larger and more complex than the three-dimensional universe we witness around us. Determining whether constants are truly constant is only the first step on a path that leads to a deeper and wider appreciation of that ultimate vista."

Organon F 27 (3) 2020: 325-345 
From this it (carefully) follows that:

ii. A law constructed in an algebraic modern formulation that is based upon a physical constant prima facia fits the definition of a 'universal law.'

Hence:

iii. In an algebraic formulated science (like ours), a physical constant can be seen, at least in practice, as an identifier of a 'universal law of nature.'

This conclusion can also be described as a philosophical principle:

iv. In an algebraic formulated science, the presence or absence of physical constants can be used in order to distinguish between universal laws and particular laws respectively, in the manner I suggested in the beginning of the paper. Put more generally, physical constants can be used as an identifier for testing the way we think of a certain phenomenon, field, or branch of science; i.e. whether a phenomenon is fundamental and universal, or whether it is local and particular.

Two qualifications are required:

The first note is that this is not a knock down argument, but rather a pragmatic argument for those trying to better understand and clarify current scientific practice. It is based on an observation regarding the appearance and absence of physical constants in physical equations, and a semantic analysis of the practical meaning of what it is to be a 'physical constant' in relation to a 'universal law.' For this reason, the principle I suggest is to be used carefully. Nonetheless, in Sections 3, I provide examples that support this principle.

The second note relates to the condition "in an algebraic formulated science." This condition is meant to emphasize that I am not arguing that physical constants are metaphysically necessary for formulating a universal law of nature. ${ }^{17}$ This is due to the fact that physical constants emerged in

17 Many discussions in current literature concern the question of why physical constants have the values they have, and what would happen if they had other values (e.g. anthropic principles), assuming, perhaps, that physical constants reveal some 
science only after the development of calculus and, in fact, it seems that the physical constants emerge when we translate a universal law (described verbally or geometrically) into an algebraic equation. Various examples demonstrate this emergence, such as the electrostatic constant in Coulomb's law, or the gravitational constant in Newtonian theory - the later will be discussed in Section 3. When a law is not expressed algebraically, there is no physical constant involved. ${ }^{18}$ This does not mean that the law is not universal. ${ }^{19}$ For example, it is in fact possible to imagine hypothetical intelligent creatures constructing an advanced science using a completely different language (a new geometry or other unknown language) that is capable of describing and explaining all the phenomena that our algebraic formulated science currently describes and explains - without the need for a single physical constant. ${ }^{20}$

deeper truth about order in the universe, or the meaning of the universe (see Smeenk and Ellis 2017). This paper, however, examines the concept of physical constants from a metaphysical neutral point of view, for it is possible that constants are simply a tool of a specific language. Even if they are only a tool of calculus, the examination is of interest given the relation of physical constants with the concept of 'law of nature.' The kind of discussion I engage in here is, as far as I know, absent from current literature.

18 In Coulomb's original book (Coulomb 1785), it is clear that like in the Principia, Coulomb's law is introduced in a descriptive manner, without a physical constant. As with the gravitational constant $(G)$, the electrostatic constant $\left(k_{e}\right)$ appears only later, when the law is formulated algebraically.

19 The law of inertia can also be given as an example of a universal law that does not have a constant. However, it is not formulated algebraically, so my principle does not apply.

${ }^{20}$ There is a view that distinguishes between dimensional and dimensionless constants. For example, according to Duff (2015), dimensional constants, such as $h, c$, $G$, $e$, or $k$, are merely human constructs whose number and values differ from one choice of units to the next. On the other hand, only dimensionless numbers such as the fine structure constant, $\alpha$, are independent of one's choice of units or measuring apparatus. Duff argues that in this sense only dimensionless constants are fundamental. However, the distinction and principle I suggest do not, in any sense, take a side in this issue. My purpose is different, and although related to the question of fundamental (or universal) laws - does not imply anything regarding the necessity nature of physical constants. 


\section{Test cases and limitations: physical constants as identifiers of universal laws}

In this section I introduce and briefly discuss two test cases in order to contextualize and further clarify my argument. The discussions presented have three purposes. The first purpose is to support my hypothesis that physical constants emerge as components in algebraic formulations of universal laws, but not in algebraic formulations of particular laws. The second purpose is to further clarify my philosophical principle that physical constants can be seen as identifiers of universal laws, and the limitations of this principle (it can be applied only in algebraic formulated science). The third is to show that in a way, my principle can help distinguish between a universal law and a particular law, while avoiding one of the long standing philosophical problems regarding laws of nature - the ceteris-paribus criterion for a generalization.

\subsection{Kepler's laws versus Newton's laws ${ }^{21}$}

Kepler's laws (Kepler [1609] 1937; [1619] 1997) were intended to describe only the solar system, whereas Newton's laws were supposed to be universal

21 It might appear that using Newtonian gravitation is not exactly the best example to a universal law, because Newton's law of gravity does not apply at speeds approaching the speed of light or in high gravitational fields, so how is it universal? A clarification is needed: It is, in fact, possible to imagine a hypothetical possible universe, in which the true physical theory is the Newtonian physics. It is clear that in that imaginary universe, Newton's law of gravitation is a truly universal law. But the truth value of a theory has nothing to do with whether it is universal or particular. In our universe, we currently refer to general relativity theory as the theory that governs the universe - but we cannot be sure that this theory is the true theory. The "true" theory of our actual universe could turn out in the future to be something completely different. However, whatever will become of fundamental physics in the future - the form of general relativity, exactly as the form of Newtonian physics, is general and universal, whether it is true or not. In other words, whether we identify a law or a theory as universal or not, has nothing to do with its truth value, but it depends on its pretense: if it was true - it is supposed to be applied to any place in the universe. Furthermore, in the case of Newton's law of gravitation - it is not a true law. It so happens that we use it, for our convenience, because in most cases 
(Smith 2008). Isaac Newton (1642-1727) devised his laws of motion and gravitation (Newton 1687) in such a way that Kepler's laws can be derived from them (Smith 2008). ${ }^{22}$

That Kepler's laws are not universal is not entirely straightforward, in a sense, because Kepler's laws are not only true for the solar system, but for any system of planets that have a gravitational force that is proportional to the inverse square of the distance between them. For example, they are true for systems of binary stars, or systems of a moon and a planet. But arguing that Kepler's laws are universal laws on this basis begs the question, for it is equal to saying that Kepler's laws are valid for every system to which the laws apply. This obviously empties the concept 'universal' of its meaning, and it is also not the universality for which we are looking.

Kepler's laws are an example of one of the long-standing disputes about laws of nature: the problem of the distinction between strict generalizations (that are usually taken to be, at the very least, true, universal statements that support counterfactual claims) and ceteris-paribus generalizations (that are usually taken to "have exceptions," to be "non-universal" or "to be ceteris paribus laws") (Reutlinger et al. 2017). The claim is that while in theory this distinction is easy enough to understand, in practice it is often difficult to distinguish strict from ceteris-paribus generalizations, because many statements with no explicit ceteris-paribus clause implicitly do have such a clause (Carroll 2016). In our example, the ceteris-paribus clause in Kepler's laws is explicit - for it is not true for systems that are not of

we are not approaching the speed of light or are found in high gravitational fields. Physicists say that the Newtonian theory is approximate at low speeds. But the term 'approximate' implies that it is not accurate - only close enough. It doesn't change the fact that the Newtonian laws do not govern our world, and if we want to be $100 \%$ accurate, we need to use other theory, and not Newton's theory.

22 Johannes Kepler (1571-1630) built upon Copernicus's work to create a much more accurate description of the solar system (Rabin 2015). The first law establishes that the orbit of a planet is an ellipse with the sun as one of the foci. According to the second law, the radius vector from the sun to a planet sweeps out equal areas in equal times, which means that the planet travels faster when closer to the sun and slower when farther from the sun. The third law (the "harmonic law") captures the relationship between the distance of planets from the sun and their orbital periods (Di Liscia 2017). 
binary stars. However, one can argue that the ceteris-paribus clause also exists in Newton's law of gravitation. For example, Cartwright (2002) is usually understood to be an advocate of ceteris paribus laws in science, including physics. More precisely, Cartwright is understood to be against the use of laws in scientific explanations, including physics, exactly because all laws in physics use ceteris paribus. Indeed, all areas of science use idealistic assumptions to simplify things: physicists describe motion on frictionless surfaces because it is too difficult to explore everything at once. A simplistic model removes confusing factors and focuses on a specific parameter. Furthermore, when it comes to testing, we test ceteris paribus laws in exactly the same way that we test laws without the ceteris paribus antecedent. However, the presence of a ceteris paribus antecedent forces us to take into account important procedures when designing experiments.

This can be somewhat confusing, since knowing that the laws of Kepler can be derived from Newton's laws makes us think, with considerable confidence, that Newton's laws are universal, while Kepler's laws are particular. Minimally, we might think that Newtonian laws are more universal than Kepler's laws. Hence, the distinction between strict generalizations and ceteris-paribus generalizations is insufficient in practice.

When converted to algebraic equations, in both Kepler's laws and Newton's laws a constant emerges. ${ }^{23}$ However, the fixed ratio in Kepler's third law does not remain steady but is different for each system. This constant depends upon the mass of the planets in the system, so when the masses are different - the constant changes. In contrast, in Newton's law of gravitation, the constant is universal, for it remains steady across space and types of physical systems no matter what their mass. This explains why the constant in Kepler's law is not a physical constant in the same sense I am dealing with in this paper. Hence, it is clear that the principle I am offering

23 This outcome demonstrates that a constant is in no way a necessary element of a universal law of nature - it is, in fact, a consequence of the translation of a universal law to an algebraic equation. For the sake of discussion, if we were constructing a fundamental science in some other language (such as verbal or geometric), perhaps there would be no need for physical constants at all in order to describe a universal law of nature. 
helps us distinguish between a universal law and a particular law while avoiding the problem of the ceteris-paribus criterion for a generalization.

\subsection{One more particular law without a constant}

Ohm's law (Ohm 1905, original work published in German 1827) is an equation $(\mathrm{R}=\mathrm{V} / \mathrm{I})$ that describes the relationship between the current through a conductor between two points in units of amperes (I), the voltage measured across the two points in units of volts $(\mathrm{V})$, and the resistance of the conductor in units of ohms (R). This law was named after the German physicist Georg Ohm (1789-1854), who carried out and described measurements of applied voltage and current through simple electrical circuits containing various lengths of wire. Ohm's law is an empirical law, that is, a generalization from many experiments that have shown that the current is approximately proportional to electric field for most materials.

According to Weber et al. (2012), in the early $20^{\text {th }}$ century, it was thought that Ohm's law would fail at the atomic scale, but in fact it was observed on a wide range of scale lengths. Moreover, Weber et al. (2012) demonstrated that Ohm's law works for silicon wires as small as four atoms wide and one atom high. However, Ohm's law is not considered a universal law, because any given material will break down (electrical breakdown) under a strong-enough electric field, and some materials of interest in electrical engineering are "non-ohmic" under weak fields (Griffiths 1999, 289). Thus, Ohm's law is a particular law of a certain class of phenomena, because it cannot show a linear, determinate and regular relation between voltage and current maintained across time. For example, in extreme temperatures the linear ratio is not maintained, and there are electrical components, such as semiconductors, for which the ratio between voltage and current is a nonlinear and unpredictable one.

I believe that this example strengthen the philosophical principle I have suggested regarding physical constants as identifiers of universal laws. Similar to the case of Kepler's laws, Ohm's law's particular status seems to follow from a non-universality of matter. Moreover, there is no physical constant in Ohm's law formulated algebraically. 


\section{Concluding remarks and further implications of the idea}

I have argued that at least in current science, there is a link between physical constants and universal laws of nature. I suggested that a physical constant can be seen as an identifier of a universal law of nature in algebraically formulated science. This observation might help us avoid problems in defining a universal versus particular law, such as strict generalizations versus ceteris paribus generalizations. It can also help clear ambiguity in the use of the term 'law' in modern physics, for it seems that we use the term 'law' rather loosely.

I started with two basic observations. The first was that in modern physics, the nomic term "law of nature" (or "universal law of nature') has practically fallen from grace. While this term is still in use, we use it without differentiating between particular and universal law. Moreover, there are many cases in fundamental physics where universal laws are not called 'laws' at all, but 'equations' or 'principles.' The second observation is that a new term emerged in the late $19^{\text {th }}$ century and early $20^{\text {th }}$ century - the 'physical constant.' ${ }^{24}$ The physical constant became so important in current, fundamental science that every new theory 'baptizes' a new physical constant. On the face of it, I have indicated that there is a superficial link between these two concepts: both laws of nature and physical constants are supposed to be universal and fundamental.

In order to test this link, I constructed a thought experiment, asking the question how science would look void of constants, for example, if we were to find that the three fundamental constants $c, h$ and $G$ are not fixed at every point in space. The answer was that it will be a science of particular laws, governing classes of phenomena. If my philosophical principle is correct, it can be used as a method to distinguish between a universal law and a particular law. Thus, physical constants can help us test the way we think of a certain phenomenon, field, or branch of science - whether a phenomenon

24 One can emphasize the technical aspect of my observation: clearly the use of physical constants emerged in science due to the use of the algebraic language of calculus and the rise of the international system of units (SI), thus there is nothing special about these quantities. However, I am not arguing here that these physical quantities are a metaphysical necessity. 
is fundamental and universal, or whether it is (only) local and particular. For example, I suggested that there is a sense in which both the 'uncertainty principle' and Schrödinger equation, though referred to in physical science as 'principle' and 'equation' respectively — in fact deserve the title 'universal law.' Nevertheless, other so called 'laws' in science are not universal laws (such as Ohm's law or Kepler's laws) but particular generalizations.

I believe that my hypothesis offers new ways to investigate these notions from a philosophical point of view. According to Carroll, "more attention needs to be paid to the language used to report what are the laws and the language used to express the laws themselves. It is clear that recent disputes about generalizations in physics and the special sciences turn on precisely these matters, but exploring them may also pay dividends on central matters regarding ontology, realism vs. antirealism, and supervenience" (Carroll 2016). In a way, this paper is doing exactly this: paying attention to the contemporary language of universal laws, which is, in our case algebraic.

A further implication of the proposition that a physical constant can be an identifier of a universal law of nature is the following hypothesis: since the rise of physical constants, they can be seen (de facto) in an algebraic formulated science as a substitute for the historical concept 'law of nature.' This might answer Caroll's (2016) question how can philosophy advance beyond the long standing disputes over laws of nature. An interesting possibility is that perhaps lawhood is not supposed to be part of the content of algebraic formulated scientific theories. In that formulation we have other, more suitable content in the form of a physical constant, which fulfills the same need for universality, stability, and fundamentality (as universal laws) - but from within the theory itself. This claim of 'replacement' is surely stronger than what I suggested here regarding the physical constants as identifiers of universal laws. If taken seriously, the claim must be developed further, because the only connection between universal laws and physical constants may be that both a universal law and a constant are universal, and that both are invariant across time and space. That connection is clearly insufficient for a stronger claim of replacement.

Lastly, the principle I suggested might also shed light upon an old controversy: can there be any special-science universal laws? If my hypothesis is correct, then the answer is basically affirmative -if a universal and 
invariant constant is rooted in the equations of a special science, its presence would imply that the law is universal and not particular.

\section{Acknowledgements}

I am grateful to Giora Hon, Yael Kedar, and Ayelet Shavit, for providing feedback on an earlier draft of this article. I thank Joseph Agassi for a helpful conversation concerning physical constants, and to Gad Freudenthal, and Raz Chen-Morris for a useful discussion about laws of nature.

\section{References}

Barrow, John D., and Webb, John K. 2005. "Inconstant constants." Scientific American 292 (6): 56-63. https://doi.org/10.1038/scientificamerican0206-64sp Carroll, John W. 2016. "Laws of Nature." The Stanford Encyclopedia of Philosophy, edited by Edward N. Zalta. https://plato.stanford.edu/archives/fall2016/entries/laws-of-nature/

Cartwright, N. 2002. "In Favor of Laws that Are Not Ceteris Paribus After All." In Ceterus paribus laws, edited by John Earman, Clark Glymour, and Sandra Mitchell, 149-63. Dordrecht: Springer. https://doi.org/10.1007/978-94-0171009-1_8

Clotfelter, Beryl E. 1987. "The Cavendish Experiment as Cavendish Knew It." American Journal of Physics 55 (3): 210-13. https://doi.org/10.1119/1.15214

Cornu, A., and Baille, J. B. 1873. "Mutual Determination of the Constant of Attraction and the Mean Density of the Earth." C. R. Acad. Sci., Paris 76: 95458.

Crane, Tim, and Mellor, D. H. 1990. "There is No Question of Physicalism." Mind 99 (394): 185-206. https://doi.org/10.1093/mind/XCIX.394.185

Dahan, Orli. 2019. "There IS a question of Physicalism." Organon F 26 (4): 54271. https://doi.org/10.31577/orgf.2019.26401

Di Liscia, Daniel A. 2017. "Johannes Kepler." The Stanford Encyclopedia of Philosophy, edited by Edward N. Zalta. https://plato.stanford.edu/archives/fall2017/entries/kepler/

Duff, Michael J. 2015. "How Fundamental Are Fundamental Cconstants?" Contemporary Physics 56 (1): 35-47. https://doi.org/10.1080/00107514.2014.980093

Fiorese, Raphaël. 2016. "Stoljar's Dilemma and Three Conceptions of the Physical: A Defence of the Via Negativa." Erkenntnis 81 (2): 201-29. https://doi.org/10.1007/s10670-015-9735-0 
Frigg, Roman. 2009. "GRW Theory (Ghirardi, Rimini, Weber model of quantum mechanics)." In Compendium of Quantum Physics: Concepts, Experiments, History and Philosophy, edited by Daniel Greenberger, Brigitte Falkenburg, Klaus Hentschel, and Friedel Weinert, 266-70. Heidelberg and Berlin: Springer. https://doi.org/10.1007/978-3-540-70626-7_81

Greene, Brian. 2003. The Elegant Universe: Superstrings, Hidden Dimensions and the Quest for the Ultimate Theory. NY: W.W. Norton \& Company.

Griffiths, David J. 1999. Introduction to Electrodynamics (3rd ed.). Prentice Hall.

Harvey, Alex, and Schucking, Engelbert. 2000. "Einstein's Mistake and the Cosmological Constant." American Journal of Physics 68 (8): 723-27. https://doi.org/10.1119/1.19534

Kepler, J. [1609]. 1937. Astronomia Nova. Pragae.

Kepler, J. [1619]. 1997. The Harmony of the World (Vol. 209). American Philosophical Society.

Lévy-Leblond, J. M. 1979. "The Importance of Being (a) Constant." In Problems in the Foundations of Physics, Proceedings of the International School of Physics 'Enrico Fermi' Course LXXII, Varenna, Italy, July 25 - August 6, 1977, edited by G. Toraldo di Francia, 237-63. Amsterdam, New York: NorthHolland.

Lizzi, Fedele, Mangano, Gianpiero, and Porzio, Alberto. 2016. "Planck's Inconstant." In Proceedings of the Corfu Summer Institute 2015. SISSA Medialab. https://doi.org/10.22323/1.263.0108

Mangano, Gianpiero, Lizzi, Fedele, and Porzio, Alberto. 2015. "Inconstant Planck's Constant." International Journal of Modern Physics A 30 (34): 1550209. https://doi.org/10.1142/S0217751X15502097

Matsas, George E., Pleitez, Vicente, Saa, Alberto, and Vanzella, Daniel A. 2007. "The Number of Dimensional Fundamental Constants." arXiv preprint arXiv:0711.4276.

Milton, John R. 1981. "The Origin and Development of the Concept of the 'Laws of Nature." European Journal of Sociology/Archives Européennes de Sociologie 22 (2): 173-95.

Newton, Isaac. 1687. Philosophiae Naturalis Principia Mathematica.

Ohm, Georg S. 1905. The Galvanic Circuit Investigated Mathematically (No. 102). D. Van Nostrand Company.

Peebles, P. J. E., and Ratra, Bharat. 2003. "The Cosmological Constant and Dark Energy." Reviews of Modern Physics 75 (2): 559.

https://doi.org/10.1103/RevModPhys.75.559

Prelević, Duško. 2017. "Hempel's Dilemma and Research Programmes: Why Adding Stances Is Not a Boon." Organon F 24 (4): 487-510. 
Rabin, Sheila. 2015. "Nicolaus Copernicus." The Stanford Encyclopedia of Philosophy, edited by Edward N. Zalta. https://plato.stanford.edu/archives/fall2015/entries/copernicus/

Reutlinger, Alexander, Schurz, Gerhard, and Hüttemann, Andreas. 2017. "Ceteris Paribus Laws." The Stanford Encyclopedia of Philosophy, edited by Edward N. Zalta. https://plato.stanford.edu/archives/spr2017/entries/ceteris-paribus/

Romer, M., and Cohen, I. Bernard. 1940. "Roemer and the First Determination of the Velocity of Light (1676)." Isis 31 (2): 327-79.

https://doi.org/10.1086/347594

Rosen, Joe. 1989. "Symmetry at the Foundations of Science." Computers $\&$ Mathematics with Applications 17 (1-3): 13-15.

Ruby, Jane E. 1986. "The Origins of Scientific 'Law." Journal of the History of Ideas 47 (3): 341-59. https://doi.org/10.2307/2709657

Shapin, S. 1996. The Scientific Revolution. Chicago and London: University of Chicago Press.

Smeenk, Christopher, and Ellis, George. 2017. "Philosophy of Cosmology." The Stanford Encyclopedia of Philosophy, edited by Edward N. Zalta. https://plato.stanford.edu/archives/win2017/entries/cosmology/

Smith, George. 2008. "Newton's Philosophiae Naturalis Principia Mathematica." The Stanford Encyclopedia of Philosophy, edited by Edward N. Zalta. https://plato.stanford.edu/archives/win2008/entries/newton-principia/

Uzan Jean-Philippe. 2011. "Varying Constants, Gravitation and Cosmology." Living Rev. Relativity 14 (2): 1-155. https://doi.org/10.12942//rr-2011-2

Weber, B., Mahapatra, S., Ryu, H., et al. 2012. "Ohm's Law Survives to the Atomic Scale." Science 335 (6064): 64-67. https://doi.org/10.1126/science. 1214319

Westphal, Volker, Rollins, Andrew M., Radhakrishnan, Sunita, and Izatt, Joseph A. 2002. "Correction of Geometric and Eefractive Image Distortions in Optical Coherence Tomography Applying Fermat's Principle." Optics Express 10 (9): 397-404. https://doi.org/10.1364/OE.10.000397

Zilsel, Edgar. 1942. "The Genesis of the Concept of Physical Law." The Philosophical Review 51 (3): 245-79. https://doi.org/10.2307/2180906 